\title{
MODÉLISATION DE LA CANOPÉE FORESTIÈRE PAR PHOTOGRAMMÉTRIE DEPUIS DES IMAGES ACQUISES PAR DRONE
}

\author{
Jonathan Lisein ${ }^{1,2}$, Stéphanie Bonnet ${ }^{1}$, Philippe Lejeune ${ }^{1}$, Marc Pierrot-Deseilligny²,3 \\ 1 : Université of Liège - Gembloux Agro-Bio Tech. Unité de Gestion des Ressources forestières et des Milieux naturels \\ 2, Passage des déportés, 5030 Gembloux, Belgique \\ 2 : Université Paris-Est (UPE), 6 et 8 avenue Blaise Pascal - Cité Descartes - Champs-sur-Marne - 77455 Marne la Vallée, France \\ 3 : Institut National de l'Information Géographique et Forestière (IGN), laboratoire LOEMI \\ 73, avenue de Paris - 94165 Saint-Mandé cedex, France
}

\begin{abstract}
Résumé
Les petits drones civils développés à des fins de cartographie rapide offrent, à une échelle locale, de nombreuses opportunités pour le suivi d'écosystèmes forestiers. Nous utilisons dans cette recherche des images acquises avec un avion sans pilote à voilure fixe afin de modéliser la surface de la canopée de peuplements feuillus. Une chaine de traitements photogrammétriques est mise en place au moyen des outils de la suite open source MICMAC. Nous comparons différentes stratégies de corrélation automatique d'images afin de déterminer le paramétrage qui permet au mieux de reconstruire les détails de la canopée. Bien que le modèle de surface photogrammétrique ne permette pas d'appréhender les petites dépressions et élévations des houppiers, nos résultats montrent que l'utilisation conjointe d'images drone et d'un modèle numérique de terrain LiDAR permet d'estimer la hauteur dominante des peuplements feuillus. Ces résultats confirment la faisabilité de modéliser l'évolution de la hauteurs des peuplements forestiers depuis une série temporelle d'images drone.
\end{abstract}

Mots clés : Modèle numérique de canopée, Drone, Photogrammétrie, Foresterie, Corrélation dense, logiciel MICMAC.

\begin{abstract}
Recent development of small unmanned aerial systems opens the door for their use in forest mapping, as both the spatial and temporal resolution of drone imagery better suit local-scale investigation than traditional remote sensing tools. An original photogrammetric workflow, based on the open source toolbox MICMAC, was set up to model the forest canopy surface from low-altitude aerial images. In combination with a LiDAR digital terrain model, the elevation of vegetation was determined after a fine co-registration of the photogrammetric canopy surface model. The investigation of different images matching strategies is performed within MICMAC and their performance in modelling the outer canopy is compared. Although photogrammetric reconstruction do not account for small peaks and gaps in the canopy surface, our results have shown the potential of drones to accurately estimate canopy height in broadleaf stands, confirming thus the feasibility of modeling height growth from UAV images time series.
\end{abstract}

Keywords : Canopy height model, Unmanned Aerial Systems, Photogrammetry, Forestry, Dense Matching, MICMAC.

\section{Introduction}

L'utilisation de drones en géomatique, ces robots volants sans pilote à bord, apparaît comme une véritable révolution pour de nombreux utilisateurs des techniques de télédétection. En effet, I'utilisation de drone pour l'acquisition d'images aériennes présente comme caractéristique novatrice, outre les avantages liés à la résolution spatiale et temporelle, le fait que l'utilisateur devient lui-même commanditaire ou même fournisseur de l'imagerie. Cette chaîne d'acquisition, de traitement et d'utilisation des images est une filière intégrée et flexible qui permet de répondre à divers besoins spécifiques. Ces spécificités se traduisent en terme d'une diversité de plateformes (à voilure fixe ou voilure tournante), de capteurs (actif, passif), de plan de vol (hauteur, recouvrement), de moment d'acquisition (conditions météorologiques, luminosité) et de période de revisite (de quelques jours à plusieurs années). En particulier, les drones petits et légers vont très probablement devenir un outil de premier plan pour les scientifiques et les environnementalistes (Koh and Wich, 2012; Anderson and Gaston, 2013). En effet, ceux-ci permettent une observation détaillée de phénomènes écologiques localisés. Un frein au développement de l'utilisation civile de drones, commerciale ou non, réside cependant en l'absence d'adéquation entre la législation et cette technologie émergente (Watts et al., 2012).

En parallèle avec l'émergence de l'utilisation des drones en cartographie, la complémentarité des techniques de photogrammétrie et de vision par ordinateur, en particulier les algorithmes d'orientation externe qui 
permettent la détermination automatique de la position et de l'orientation des prises de vues d'une collection d'images se recouvrant ${ }^{1}$, a initié un regain d'intérêt pour la photogrammétrie. L'omniprésence de la photographie numérique et l'amélioration continue de la puissance de calcul des ordinateurs rendent aujourd'hui les techniques de photogrammétrie attrayantes et concurrentielles (Pierrot-Deseilligny and Clery, 2011b). Les chaînes de traitement photogrammétrique présentent un degré d'automatisation de plus en plus élevé, permettant la reconstruction tridimentionnelle depuis une masse d'images issues d'appareils photographiques compacts non calibrés.

L'objectif de la présente recherche est d'observer la canopée au moyen d'images acquises par drones et d'en déduire des indices de structure des peuplements forestiers, en particulier la structure verticale. La mesure de la hauteur de la végétation est une information qui intéresse autant le gestionnaire forestier que le scientifique œuvrant dans ce domaine. La hauteur de la canopée est un indicateur de maturité des peuplements très usité en gestion, aussi bien pour l'estimation de la biomasse que pour la planification des interventions sylvicoles. De manière plus générale, la hauteur de la canopée est utilisée pour la modélisation de nombreux phénomènes liés aux écosystèmes forestiers. De manière usuelle, c'est le LiDAR $^{2}$ aérien qui est utilisé pour capturer finement l'information de la hauteur de la canopée et du sol. Les émissions de rayons lasers d'un LiDAR aéroporté percutent la canopée forestière ainsi que le sol et réfléchissent une partie de l'énergie émise vers le capteur, permettant de cette manière une mesure de la hauteur de la végétation (Bonnet et al., 2013). C'est la capacité des émissions LiDAR de pénétrer le couvert forestier qui confère à ce capteur actif la possibilité de mesurer directement la hauteur de la canopée. La cartographie de la hauteur de la végétation est simplement effectuée par la soustraction de l'altitude du sol (Modèle Numérique de Terrain, MNT) à l'altitude de la végétation (Modèle Numérique de Surface de la canopée, MNS).

Le résultat est un Modèle Numérique de Canopée (MNC), encore désigné sous l'appellation de Modèle Numérique de Hauteur (MNH). Les techniques de photogrammétrie numérique, elles, permettent la modélisation de la surface de la canopée, soit uniquement le MNS. L'utilisation conjointe d'un MNS photogrammétrique avec un MNT LiDAR permet la mesure de la hauteur de la végétation en combinant les avantages des deux méthodes d'acquisition (LiDAR et photogrammétrie aérienne), notamment les avantages en terme de résolution et de temps de revisite des images aériennes

1. "structure from motion" en anglais, voir (Snavely et al., 2008).

2. acronyme de Light Detection and Ranging.
(White et al., 2013). En effet, l'utilisation du LiDAR à haute densité est limitée par son prix d'acquisition qui reste substantiellement plus élevé que celui d'une prise de vue aérienne. Dès lors, une opportunité intéressante est de mettre régulièrement à jours un MNC LiDAR avec un MNS photogrammétrique, afin de tirer parti et de la précision du MNT LiDAR et du temps de revisite des acquisitions par drone.

Cependant, le calcul des éléments de l'orientation des caméras ainsi que la corrélation dense en milieu forestier sont reconnus comme étant difficilement réalisables (Véga and St-Onge, 2008; Baltsavias et al., 2008). En effet, de nombreuses propriétés de la végétation entravent la bonne réalisation des traitements photogrammétriques. Les changements brusques d'altitude entre une couronne d'arbre et le sol ainsi que la micro-topographie de la canopée entrainent de multiples occlusions sur les images aériennes. La texture répétitive des feuilles, des branches et des couronnes d'arbre rendent difficile aussi bien la détermination automatique de points de liaison que le processus de corrélation dense. Certaines recherches ont néanmoins démontré que ces difficultés peuvent être partiellement surmontées et que la génération par photogrammétrie de modèle de surface de la canopée aboutit à des résultats d'une résolution et d'une précision suffisante pour les applications forestières (Véga and St-Onge, 2008, 2009).

\section{Objectifs}

La présente recherche vise à utiliser des images acquises par drones en combinaison avec un modèle numérique de terrain LiDAR pour modéliser la hauteur de la canopée forestière. Les traitements d'images drone sont effectués avec la chaîne photogrammétrique open source de I'IGN ${ }^{3}$, MICMAC (Pierrot-Deseilligny, 2014). La comparaison de différentes approches de corrélation et la mise au point d'une chaine de traitements adaptée et opérationnelle sont les motivations sous-jacentes de cette recherche.

Le choix de l'utilisation de MICMAC est justifié, outre par sa gratuité et son caractère open source, par son haut degré de paramétrisation permettant d'appréhender le matériel photographique de peuplements forestiers acquis par drone. La suite MICMAC comprend un ensemble d'outils en ligne de commande. Certains outils sont hautement paramétrables : leur utilisation se fait via l'utilisation de fichiers $X M L$ regroupant l'ensemble du paramétrage. D'autres outils sont dit simplifiés, par opposition aux outils complexes. Ce sont des outils pour lesquels la plupart des paramètres ont une valeur par défaut. L'utilisation des outils simplifiés se fait au moyen

3. L'Institut National de l'Information Géographique et Forestière. 
d'une ligne de commande contenant l'ensemble des paramètres. La génération et la validation du MNC sont présentées dans l'article (Lisein et al., 2013). Le présent document se focalise plus en détails sur la description des traitements photogrammétriques, notamment les différentes approches de mise en correspondance qui ont fait l'objet d'investigations. D'une part, la mise en correspondance est effectuée en géométrie terrain au moyen de deux outils, un outil simplifié (Malt) et un outil complexe (Micmac). D'autre part, la corrélation est effectuée en géométrie image au moyen de l'outil MMByP.

\section{Matériel et Méthode}

\subsection{La zone d'étude, le vecteur et le capteur d'acqui- sition}

La zone d'étude est un massif forestier situé dans la commune de Felenne en Belgique, à cheval sur les lieux-dits petit Houdron et les Rolisses. Les 200 ha de forêt sont composés majoritairement de peuplements feuillus mélangés à prédominance de chênes indigènes (Quercus robur L. et Quercus petraea (MATT) Liebl.). Ces peuplements sont issus de la conversion de vieux taillis et de vieux taillis sous futaie et sont caractérisés par une structure irrégulière. Les peuplements résineux, représentant $25 \%$ du massif forestier, sont pour la plupart des pessières équiennes. Une campagne de mesures d'inventaire s'est déroulée en 2012 afin d'estimer la hauteur dominante en vue de confronter le modèle numérique de canopée photogrammétrique à des données terrain (voir (Lisein et al., 2013) pour de plus amples détails concernant la validation du MNC).

Le drone utilisé est un drone à voilure fixe de type léger, le Gatewing $X 100^{4}$ (Figure 1). Le Gatewing X100 est vendu "prêt à voler", pèse un peu moins de $2 \mathrm{~kg}$ pour 1 mètre d'envergure et est doté d'une propulsion électrique. Le décollage s'effectue au moyen d'une rampe de lancement à élastiques. Sa vitesse de croisière est de $80 \mathrm{~km} / \mathrm{h}$ et son autonomie de vol est de 40 minutes. Les vols sont entièrement automatisés, du décollage à l'atterrissage (atterrissage sur le ventre). La préparation de la prise de vue aérienne s'effectue avec une station de contrôle au sol en définissant les paramètres de vol suivants : délimitation de la zone rectangulaire à couvrir, hauteur de vol (entre 100 et 750 mètres au dessus du niveau du sol), recouvrement des images (le recouvrement latéral est de même valeur que le recouvrement longitudinal), ainsi que la direction et le sens du décollage et de l'atterrissage. Son système de pilote automatique est équipé d'une centrale inertielle (gyroscopes et baromètre) ainsi que d'un GPS atteignant une précision de quelques mètres.

Le capteur embarqué est un appareil digital compact de modèle Ricoh GR3 (capteur photographique CCD de

\footnotetext{
4. http ://uas.trimble.com/X100.
}

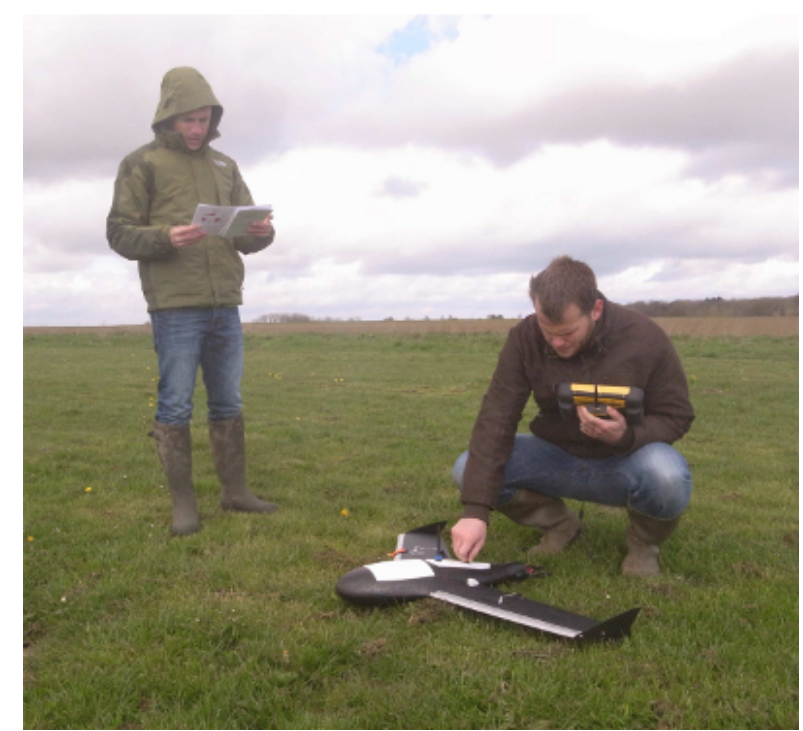

FIGURE 1 : Les deux pilotes à distance récupèrent le drone Gatewing X100 après un atterrissage. Le pilote de gauche tient la liste de vérification des opérations et le pilote de droite effectue les opérations, tantôt sur le drone, tantôt sur la station de contrôle au sol qu'il tient à la main.

10 megapixels et distance focale équivalente en $35 \mathrm{~mm}$ de $28 \mathrm{~mm}$ ). Cet appareil est modifié pour les acquisitions dans le proche infra-rouge. L'appareil est employé en mode manuel et la sensibilité ISO (réglé à 200) ainsi que le temps de pose (réglé à 1/2000) sont définis juste avant le décollage. Le déclenchement de l'appareil est effectué par le pilote automatique sur base de la position de l'avion. L'acquisition s'est déroulée en un vol unique durant la période de végétation, le $1^{\text {er }}$ août 2012 entre 16 et 17 heure de l'après midi. L'altitude de vol est fixée à 225 mètres au dessus du point de décollage avec un recouvrement de $75 \%$. La résolution spatiale en résultant est de l'ordre de $7,6 \mathrm{~cm} /$ pixel et le rapport $B / H^{5}$ est de 0,3. La zone couverte en stéréoscopie représente plus de 200 ha. Le tableau d'assemblage est composé de 441 images de prises de vue légèrement oblique (figure 2). L'orientation des prises de vue successives varie fortement en fonction de l'attitude du drone dont la stabilité est mise à mal par les conditions de vent : la configuration soleil-objet-capteur est très différente entre chaque prise de vue (Aber et al., 2010). Du flou est observable sur presque $80 \%$ des images, dû à un effet de filé (flou engendré par le déplacement du drone) et aux vibrations provoquées par le moteur.

Pour modéliser la hauteur de la végétation à partir

5. $B$ étant la base, soit la distance entre deux prises de vue successives, et $H$ étant la hauteur de vol. le ratio $B / H$ est un paramètre renseignant l'angle d'intersection des rayons homologues : plus l'angle d'intersection des rayons homologues est élevé, plus la corrélation est précise, mais plus l'angle d'intersection des rayons homologues est faible, plus la corrélation est robuste. 
d'un MNS photogammétrique, il est nécessaire de disposer d'une information sur l'altitude du sol sous la canopée. Le MNT utilisé dans ce but provient d'un vol LiDAR effectué en juin 2011. Cette acquisition a généré un nuage de points de haute densité, de l'ordre de 13 points $/ \mathrm{m}^{2}$. L'extraction d'un MNT de $0,5 \mathrm{~m}$ de résolution depuis le nuage de points a été effectué par le prestataire de service. Sa précision est estimée à $0,14 \mathrm{~m}$ de RMSE en altimétrie par comparaison avec des points de contrôle au sol. Le nuage de points LiDAR a également permis d'extraire un MNS et un MNC, qui sont utilisés pour la validation de la chaîne de traitements photogrammétriques développée lors de cette recherche.

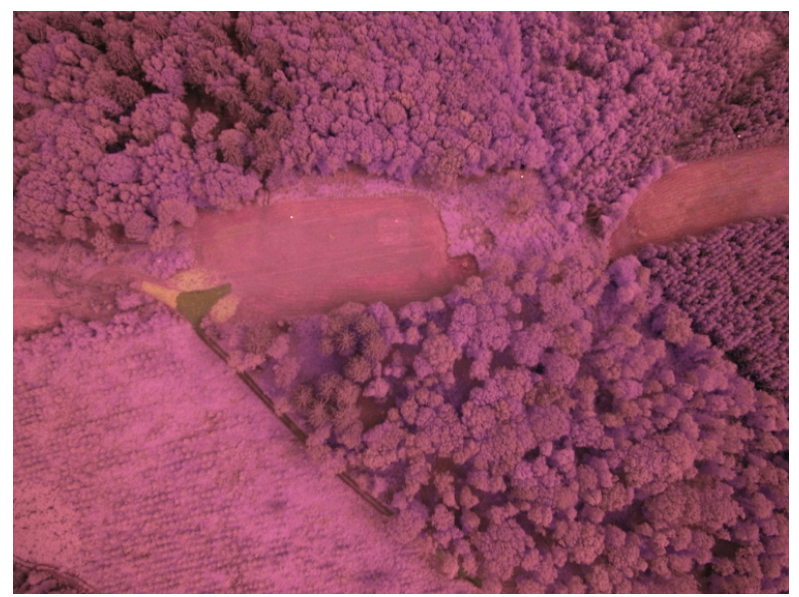

FIGURE 2 : Une des 441 images dans le proche infra-rouge acquises sur le massif forestier de Felenne.

\subsection{Le calcul des éléments d'orientation du bloc d'images}

\subsubsection{La génération des points homologues}

La génération automatique des points homologues s'effectue au moyen de l'algorithme SIFT de Lowe (2004). L'outil de la suite MICMAC interfaçant SIFT est Tapioca. Avec Tapioca, différentes stratégies permettent de calculer les points de liaison. Ces stratégies permettent de déterminer les couples d'images pour lesquels s'effectue le calcul des points homologues. L'approche basique est de sélectionner tous les couples possibles au sein d'un bloc de $n$ images (soit $n *(n-1) / 2$ couples d'images), mais cette solution requiert un temps de calcul plus long et n'est pas adaptée à un bloc d'images volumineux. Pour une acquisition par drone avec GPS embarqué, l'approche jugée la plus adéquate consiste à sélectionner pour chaque image, toutes les associations de cette image avec ses plus proches images voisines. Pour ce faire, l'outil Oriconvert, au départ des données d'orientations externes initiales, calcule par triangulation de Delaunay les voisins de chaque image. Le résultat est enregistré sous forme d'un fichier XML contenant, pour l'ensemble du bloc d'images, tous les couples résultant. Cette liste de couples d'images est ensuite utilisée par Tapioca pour le calcul des points homologues. Étant donné la faible netteté des images aériennes de Felenne et les fortes distorsions dues aux changements du micro-relief forestier et à la prise de vue, il apparaît inopportun d'effectuer le calcul des points homologues à pleine résolution : les images (de $3648 \times 2736$ pixels) sont donc ré-échantillonnées par Tapioca en vue d'obtenir des images d'une largeur de 2000 pixels.

\subsubsection{Le calcul de l'orientation absolue finement recalée sur le MNT LiDAR}

La détermination des éléments de l'orientation du bloc d'images, à savoir l'orientation externe (position $X$, $Y, Z$ et orientation omega, phi, kappa) et l'orientation interne (calibration de la caméra), s'effectue au moyen de la méthode de compensation par faisceaux implémentée dans l'outil simplifié Tapas (Pierrot-Deseilligny and Clery, 2011a). La compensation par faisceaux utilise les équations de colinéarité, qui relient les coordonnées images $(u, v)$ des observations de points homologues aux coordonnées modèles $(x, y, z)$, pour résoudre ce système d'équations non-linéaires surabondant. Cette méthode d'ajustement nécessite, outre les observations des points homologues, de disposer d'une valeur initiale des paramètres d'orientation interne. Une calibration initiale de l'appareil photographique est calculée préalablement à l'acquisition avec Tapas en utilisant une dizaine d'images convergentes d'une scène terrestre présentant de la profondeur et de la texture, comme par exemple un coin de bâtiment. Le calcul de l'orientation relative du bloc d'images, illustré à l'étape (1) de la chaîne de traitements en figure 3 , s'effectue ensuite en autocalibration. Le modèle de distorsion utilisé est le modèle de Fraser, utilisant 3 coefficients pour appréhender la distorsion radiale (Pierrot-Deseilligny, 2014).

Le calcul de l'aérotriangulation sans autres observations ou contraintes que celles des points homologues présente un résultat caractérisé par des distorsions nonlinéaires. L'inspection visuelle du modèle d'aérotriangulation du bloc d'images de Felenne nous a en effet permis de constater des distorsions paraboliques, créant un effet de bol sur le chantier. L'ajout de contraintes dans la compensation permet de corriger ces distorsions non-linéraires. Nous utilisons pour ce faire les observations du GPS embarqué (notés Centres figure 3) dans une nouvelle compensation par faisceaux, opérée avec Apero (étape (2)). Bien que ces mesures GPS soient peu précises et entachées d'un biais dans le sens du vol, biais provoqué par un décalage entre l'enregistrement de la position GPS et le moment de la prise de la photographie, la compensation par faisceaux avec les valeurs approximatives des centres optiques des prises de vue permet de supprimer ces distorsions non-linéaires. L'orientation en résultant est géoréférencée dans un système de coordonnées cohérent avec les données LiDAR (dans notre cas, le système de projection Belge Lambert 72). 


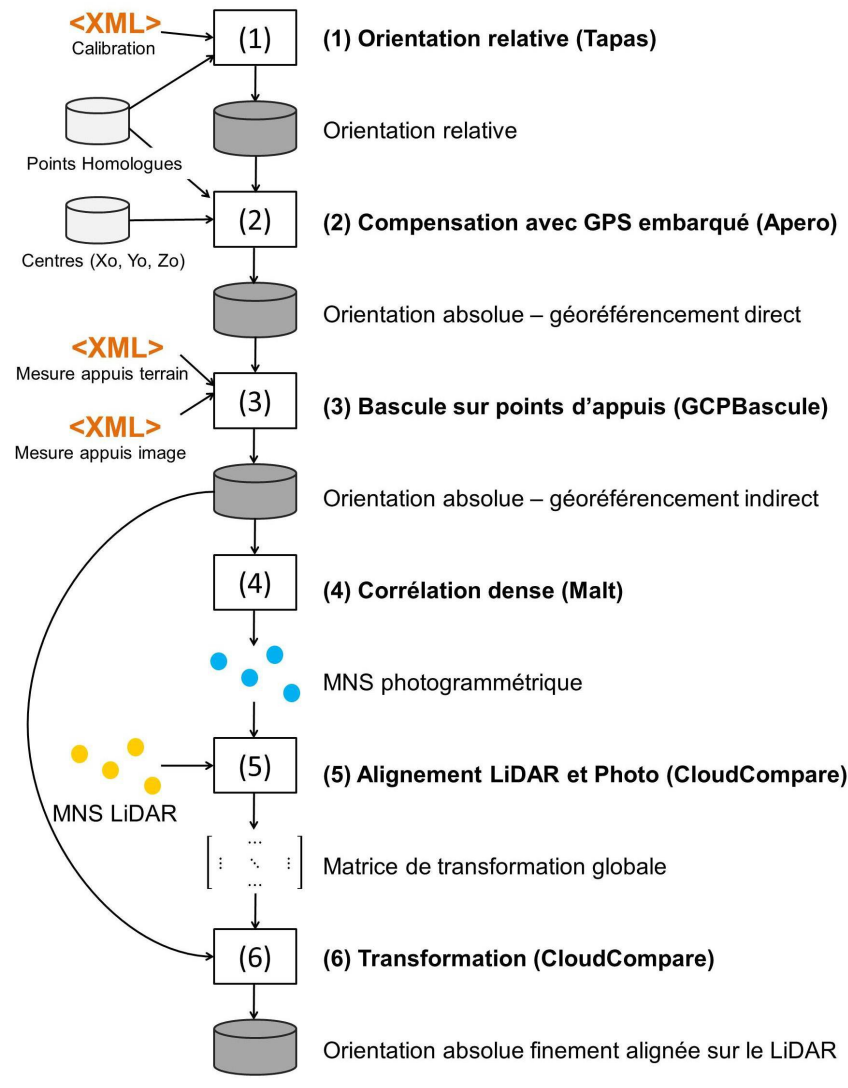

FIgURE 3 : La chaîne de traitement du calcul de l'orientation absolue recalée sur le MNS LiDAR. Légende : traitement : $\square$ - base de donnée : $\bigcirc$ - fichier xml : <xML> - matrice : $\left[\begin{array}{l}: \\ \vdots\end{array}\right]$. nuage de point : $\bullet$.

Les mesures précises de hauteurs depuis un MNC photogrammétrique sont envisageables pour autant que l'enregistrement conjoint ${ }^{6}$ du MNS photogrammétrique avec le MNT LiDAR soit satisfaisant (Baltsavias et al., 2008). Afin d'assurer une bonne cohérence entre le géoréférencement du bloc d'images et celui du MNT LiDAR, nous utilisons un algorithme de recalage de modèles $3 \mathrm{D}$ de type Iterative Closest Point. Le calage des 2 nuages de points, d'une part le MNS LiDAR et d'autre part le MNS photogrammétrique, permet un enregistrement conjoint du MNS photogrammétrique avec le MNT LiDAR. L'algorithme de recalage utilisé détermine par itération les 3 rotations et les 3 translations (la transformation globale) qui permettent de minimiser la distance entre les deux nuages de points (EDF R\&D Telecom ParisTech, 2014). Les deux modèles doivent donc préalablement être à la même l'échelle. Hors l'analyse des résidus sur la position GPS des centres dans la compensation en étape (2) nous a permis de constater que l'échelle du modèle est sous-estimée. Afin de mettre le modèle d'aérotriangulation à l'échelle, une bascule sur des points d'appuis est effectuée. La position de six points d'appuis

6. co-registration en anglais. (correspondant à des carrefours et marquages routiers) est relevée sur les images drone (mesures notées $\mathrm{Me}$ sure appuis images sur la figure 3 ) et leur position sur le terrain (mesures notées Mesure appuis terrain sur la figure 3) est déterminée depuis les orthophotographies de 2009 de la région wallonne $(0,25 \mathrm{~m}$ de résolution) pour la planimétrie et depuis le MNS LiDAR pour l'altimétrie. La bascule sur les points d'appuis s'effectue avec l'outil GCPBascule, qui détermine la transformation rigide de 7 paramètres ( 3 translations, 3 rotations et 1 mise à l'échelle du modèle) et permet un géoréférencement d'une précision de l'ordre de $50 \mathrm{~cm}$ (résidus des points d'appuis).

Ensuite, une corrélation dense avec l'outil simplifié Malt est réalisée en vue de générer un MNS photogrammétrique qui est ensuite recalé sur le MNS LiDAR (étapes (4) et (5) de la figure 3). Cette transformation est appliquée à l'orientation absolue obtenue précédemment pour obtenir une orientation absolue finement alignée sur le MNS LiDAR (étape (6)).

\subsection{Les stratégies de corrélation dense}

Les techniques de mise en correspondance automatique d'images se basent sur un indice de similarité des images, par exemple l'indice de corrélation croisé normalisé, pour apparier ensemble plusieurs vignettes de corrélation aux sein d'une collection d'images.

MICMAC, acronyme de Multi-Images Correspondances, Méthodes Automatiques de Corrélation, se base sur une méthode de corrélation d'images en multi stéréoscopie et utilise une stratégie multi-résolution (Pierrot-Deseilligny and Paparoditis, 2006). Cette approche multi-résolution, qui est à la fois plus rapide et plus robuste, consiste à effectuer successivement l'appariement pour différents niveaux de résolution. La corrélation est effectuée de manière successive sur une pyramide d'images : à la plus basse résolution, une solution est calculée en explorant un grand intervalle d'incertitude. La géométrie calculée est ensuite utilisée comme solution initiale pour l'appariement à un niveau de résolution supérieur, et ainsi de suite jusqu'à l'obtention d'un modèle très résolu.

La stratégie de corrélation est définie comme étant la séquence hiérarchique de mise en correspondance ainsi que le paramétrage de l'algorithme de corrélation y étant associé. La détermination d'une stratégie de corrélation optimale pour la modélisation de la canopée forestière depuis des images drone a nécessité une approche itérative d'essais-erreurs. Étant donné que la stratégie de corrélation dépend des caractéristiques des images ainsi que de celle de la scène, nous commençons par rappeler brièvement les spécificités de notre chantier. Les images acquises par drone présentent de nombreuses distorsions dues à la perspective et au relief. La redondance est néanmoins élevée, avec de très hauts recouvrements entre images. La canopée forestière de peuplements feuillus apparaît, vue du ciel, comme une collection de couronnes d'arbre de forme et de taille variables. La texture répétitive de la canopée, le mouvement (entre deux 


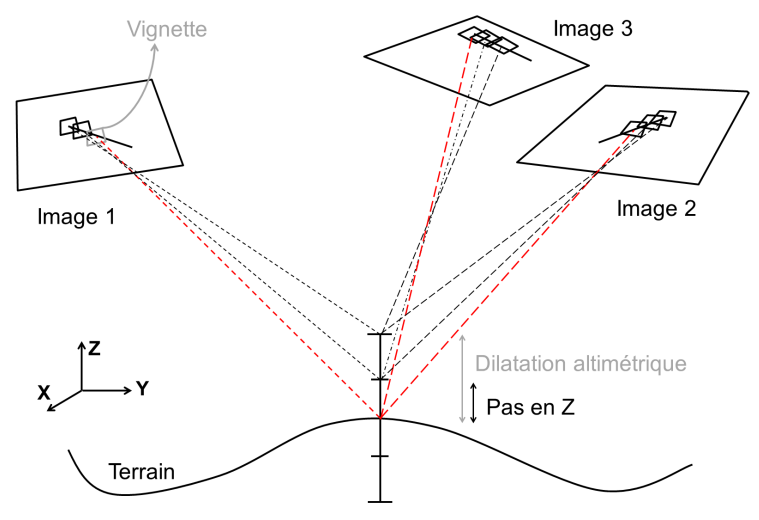

FIGURE 4 : Corrélation multi-stéréo en géométrie terrain : pour chaque position $(X, Y)$ dans la géométrie terrain, l'altitude $Z$ est recherchée le long d'un intervalle d'incertitude par la mise en correspondance des vignettes des $n$-images.

prises de vue) des branches et des feuilles dû au vent, les ombres portées, les multiples occlusions et la structure étagée du feuillage et des branches vont être autant d'éléments qui rendent la corrélation automatique en milieu forestier un véritable défi (Baltsavias et al., 2008). Ces éléments sont d'autant plus délétères à la corrélation automatique que la résolution spatiale des images acquises est élevée. Les ruptures de pente entre deux couronnes d'arbre, entre deux peuplements ou entre une clairière et les arbres avoisinants vont être déterminantes pour le paramétrage de la corrélation. Ces changements verticaux abrupts vont se traduire par un haut coefficient de dilatation altimétrique pour permettre de couvrir un grand intervalle d'incertitude allant du sol au sommet de la canopée (voir figure 4). La micro-topographie de la canopée forestière présente de fines dépressions et élévations, ce qui a orienté notre choix concernant le paramètre de régularité de la surface. Le terme de régularisation de l'algorithme de corrélation, terme qui traduit la connaissance a priori du degré de lissage de la surface à reconstruire, est ainsi réglé à une valeur très faible. Au vu de la qualité des images et des exigences du MNC en terme de résolution, la corrélation est effectuée jusqu'à une résolution de $1: 4$ de la résolution des images brutes, soit une résolution de $30 \mathrm{~cm}$.

Trois outils de la suite MICMAC sont utilisés pour la corrélation et leurs résultats sont ensuite évalués. L'outil simplifié de mise en correspondance Malt avec la stratégie prédéfinie de corrélation ortho permet le calcul d'un modèle numérique de surface en géométrie terrain (figure 4) (ou espace object, Paparoditis et al. (2000)). L'outil simplifié MMByP effectue pour sa part la corrélation en géométrie image (ou espace image, Pénard et al. (2006)) pour chaque paire d'images consécutives (figure 5). Les cartes de profondeurs résultant de l'appariement de chaque paire sont ensuite basculées en géométrie terrain et fusionnées pour délivrer un MNS couvrant l'entièreté du chantier. Enfin, l'outil complexe Micmac est utilisé en géométrie terrain

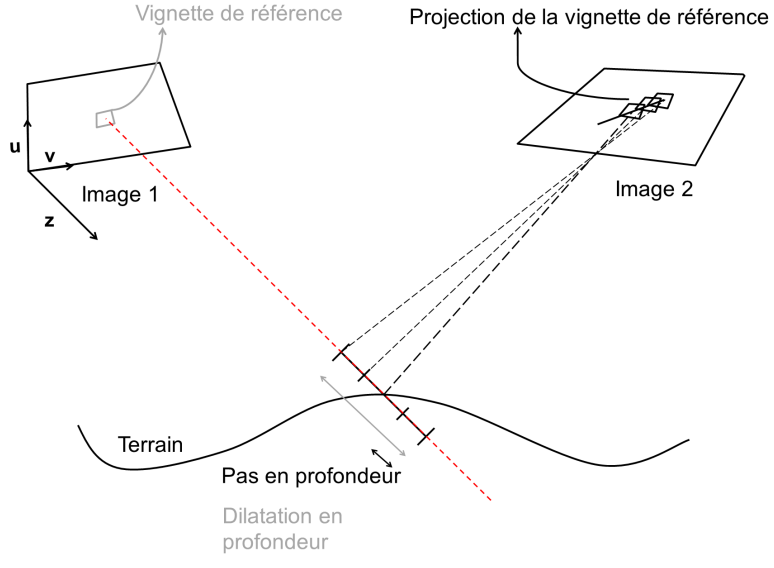

FIGURE 5 : Corrélation stéréo en géométrie image avec MMByP : pour chaque position $(u, v)$ dans la géométrie de l'image maitresse, la profondeur ( $z$ ) est recherchée le long d'un intervalle d'incertitude par la mise en correspondance des 2 images.

avec un intervalle d'incertitude très élevé et avec un facteur de régularisation très bas $(0,003)$.

Les approches de corrélation en géométrie terrain et en géométrie image sont illustrées en figures 4 et 5 respectivement.

De manière générale, la corrélation en géométrie terrain convient mieux que la corrélation en géométrie image pour des prises de vues aériennes traditionnelles, c'est à dire à haute altitude. En effet, la corrélation en géométrie terrain produit une carte unique du relief couvrant toute la zone du bloc d'images vu en stéréo. La corrélation en géométrie image, quant à elle, requiert une chaîne de traitement plus complexe. Elle délivre une carte de profondeur par images, ce qui nécessite une étape supplémentaire de fusion des cartes de profondeurs. Pour des prises de vues rapprochées telles que celles utilisées en photogrammétrie terrestre, la corrélation en géométrie images prend tout son sens, car le résultat n'est plus nécessairement une carte mais un assemblage des nuages de points. Une carte unique ne pourra en effet pas restituer la géométrie d'une statue, par exemple. Les deux géométries de corrélation, image et terrain, sont utilisées dans cette recherche, car les blocs d'images drones sont souvent à mi-chemin entre une prise de vue aérienne et une prise de vue rapprochée, et ce d'autant plus que le drone ait volé à basse altitude.

\subsection{Utilisation du modèle numérique de canopée pour la prédiction de la hauteur dominante}

La prédiction de variables forestières au moyen d'inventaire multi-source s'effectue communément via l'utilisation de modèles de régression liant un attribut forestier avec les informations acquises par télédétection (Næsset, 2002). Les modèles numériques de canopée permettent une mesure de la structure spatiale des peuplements et peuvent avantageusement être utilisés dans 
des inventaires à deux phases. Parmi les indicateurs de structure, la hauteur dominante d'un peuplement forestier est l'indice de maturité le plus usité en gestion des forêts, surtout dans le cas des peuplements réguliers. La hauteur dominante correspond à la hauteur moyenne arithmétique des 100 plus gros bois à l'hectare (Assman (1959) selon Rondeux (1999)), et est représentative de la hauteur moyenne des arbres dominants d'un peuplement. La hauteur dominante, en combinaison avec l'âge du peuplement pour des peuplements équiens et avec une autre caractéristique dendrométrique (tel que la circonférence) en peuplement irrégulier (Otoul, 1978), est en effet utilisée pour estimer la productivité d'un peuplement. Lors d'une étude pionnière sur l'utilisation des drones en foresterie, Dandois and Ellis (2013) ont prédit la hauteur dominante de peuplements feuillus réguliers avec une variable explicative extraite d'un MNC drone avec une précision de l'ordre de 3,2 mètres (erreur moyenne quadratique). Leur modèle présente un coefficient de détermination de $84 \%$. Le MNC photogrammétrique de Felenne a également servi à l'établissement de modèles prédictifs de la hauteur dominante. La campagne de mesures des caractéristiques des peuplements via des techniques appropriées d'inventaire par échantillonnage (voir Lejeune et al. (2008) à ce sujet) s'est déroulée en automne 2012 et a porté sur un total de 36 placettes d'inventaire en peuplement feuillus. Les unités d'échantillonnages sont des placettes dites à nombre de bois minimum; leur surface varie en fonction de la densité des arbres, l'objectif étant de mesurer un minimum de 20 arbres par placettes. Cette technique permet ainsi de garder l'effort d'échantillonnage indépendant de la densité (voir Lisein et al. (2013) pour de plus amples détails). Une série d'indices statistiques ${ }^{7}$ descriptifs du MNC calculés sur la zone de chaque placette d'inventaire ont servi à la modélisation de la hauteur dominante via des modèles de régression linéaire à une et à deux variables explicatives. La sélection des variables explicatives s'est effectuée par régression pas-à-pas de type best subset à l'aide du logiciel $[R]([R]$ Development Core Team, 2011).

\section{Résultats et Conclusion}

\subsection{Acquisitions par drone}

Les acquisitions par drone ont répondu à nos besoins en terme de flexibilité d'utilisation : outre le vol fructueux présenté dans ce document, de très nombreux vols ont été effectués sur Felenne à divers moments de la période de végétation dans le cadre de recherches connexes sur la discrimination des essences forestières. Néanmoins, bien qu'en Belgique l'octroi d'autorisations légales pour l'utilisation de drones soit facilité dans le cadre de recherche scientifique, les contraintes législatives relatives aux opérations avec un avion sans pilote restent un frein
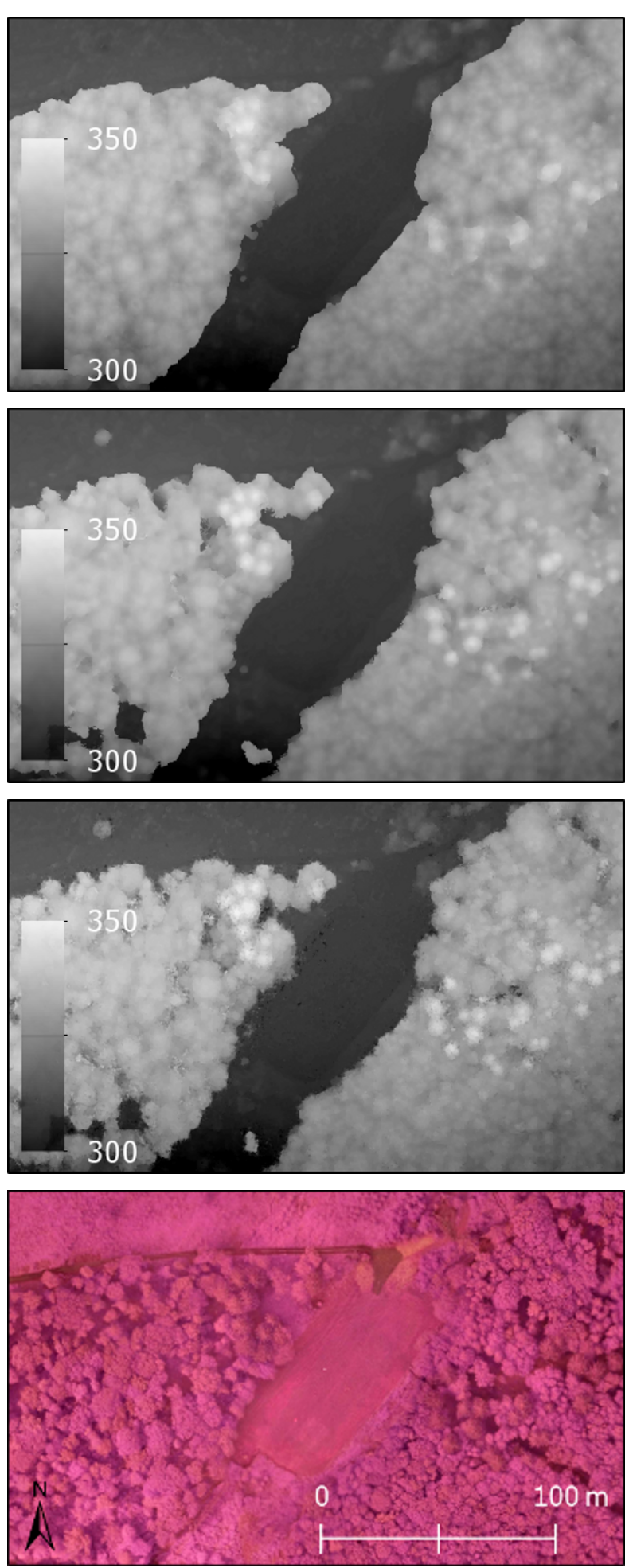

FIGURE 6 : Surface de la canopée forestière reconstruite au moyen de différentes stratégies de corrélation (de haut en bas) : Malt, MMByP et Micmac. Les niveaux de gris représentent l'altitude [m] de la canopée et du sol. En bas : une orthophotomosaique fausse couleur.

\footnotetext{
7. metric en anglais.
} 
important au déploiement rapide de cette technologie. La constitution d'un dossier de demande d'autorisation de vol et le délai de l'octroi de cette autorisation peut en effet nécessiter plusieurs semaines. Outre cet aspect, force est de constater que la stratégie d'acquisition des images par drone reste trop souvent mal adaptée aux objectifs : tout est mis en œuvre pour effectuer les traitements d'images les plus appropriés à la génération d'un modèle numérique de canopée, mais trop peu d'efforts sont mis en œuvre pour mettre en place un plan de vol permettant une prise de vue optimale. À ce niveau, le logiciel de planification de vol du Gatewing X100 a montré ses limites par son incapacité à planifier des vols avec une configuration plus personnalisée en terme de points de passage (waypoint en anglais), d'altitude de vol et de recouvrement. La mise au point d'une stratégie d'acquisition appropriée, en envisageant des plans de vol avec plusieurs niveaux d'altitude ou encore avec plusieurs capteurs optiques en vue de minimiser les occlusions, doit faire l'objet de futures recherches (Dandois and Ellis, 2013).

\subsection{Modélisation de la canopée par photogrammé- trie}

Le calcul de l'orientation absolue par aérotriangulation avec les outils de la suite MICMAC s'est déroulé avec succès pour 439 des 441 images du bloc complet : l'orientation a échoué pour deux images en bordure du bloc ne présentant pas suffisamment de points homologues. Le recalage du modèle $3 D$ photogrammétrique avec le MNS LiDAR par Iterative Closest Point a abouti à une distance entre les deux nuages de points de $0,48 \mathrm{~m}$ en moyenne (biais), avec un écart-type de 0,44 $\mathrm{m}$ (précision). Cet enregistrement conjoint du MNS photogrammétrique avec le MNS LiDAR, et donc de manière sous-jacente avec le MNT LiDAR, est satisfaisant en terme de précision.

L'inspection visuelle des résultats de la corrélation dense effectuée avec les deux outils simplifiés Malt et MMByP et l'outil complexe Micmac suggère que la qualité générale du modèle de surface de la canopée est en accord avec les recherches antérieures sur ce sujet (Véga and St-Onge, 2008; Baltsavias et al., 2008; Dandois and Ellis, 2013). Les MNS produits avec Malt et MMByP sont cependant caractérisés par une simplification plus importante de la surface de la canopée, simplification qui se traduit par un lissage plus important et donc par la non-reconstruction des petites dépressions et élévations intra et inter-houppiers. De plus, certains arbres isolés sont complètement absents du MNS généré avec Malt, bien que ces arbres soient présents sur les images individuelles (voir arbres isolés en figure 6).

Le MNS généré avec Micmac semble visuellement plus bruité mais restitue fidèlement les petites trouées entre couronnes d'arbres, comme l'illustre la figures 6 . En outre, la comparaison de ce modèle de surface avec le MNS LiDAR, représenté à la figure 7 , illustre bien

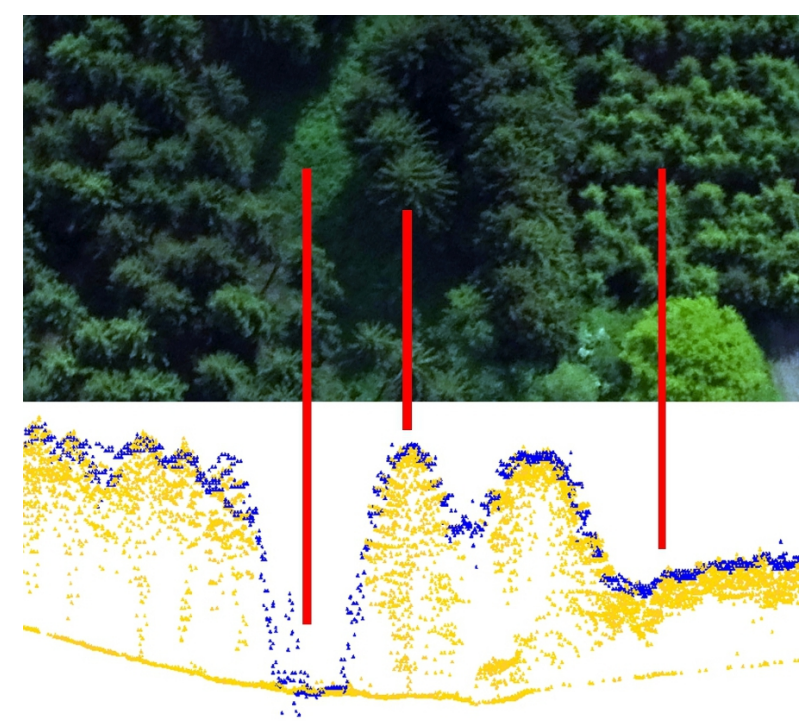

FIgURE 7 : Comparaison visuelle par transect du modèle de surface de canopée photogammétrique (points bleus) et du nuage de point LiDAR haute densité (points jaunes). Une image aérienne en couleur issue d'un autre vol drone est ajoutée en haut pour faciliter l'interprétation du transect dans les deux nuages de points.

quelques uns des avantages et des inconvénients respectifs de la photogrammétrie et du LiDAR : densité constante et élevée du MNS photogrammétrique, lissage du relief par le MNS photogrammétrique par rapport au LiDAR, capacité de pénétrer la canopée du LiDAR et d'enregistrer plusieurs retours, présence de bruit dans le MNS photogrammétrique, etc. (White et al., 2013). Ce MNS généré avec Micmac est couplé au MNT LiDAR pour la création du modèle numérique de hauteur. La comparaison approfondie de ce MNC photogrammétrique avec le MNC LiDAR est renseignée plus en détails dans un article antérieur (Lisein et al., 2013). La mise au point d'une stratégie de corrélation automatique avec les outils de la suite MICMAC a nécessité de nombreux tests. Certes, la flexibilité de ces outils, qui permettent si nécessaire un réglage personnalisé d'un nombre important de paramètres et qui génèrent un nombre très important de produits intermédiaires, est en adéquation avec les besoins de cette recherche. II nous faut néanmoins préciser que la courbe d'apprentissage des outils complexes de la suite MICMAC est rude. Leur utilisation est donc plus appropriée à des fins pédagogiques ou scientifiques qu'aux utilisateurs ponctuels souhaitant obtenir une orthophotomosaique ou un modèle numérique de surface depuis un jeu d'images. Cependant, la robustesse de la corrélation en géométrie image implémentée dans l'outil actuellement en développement MMByP, robustesse due au $B / H$ moindre des couples d'images successives et à la limitation du diachronisme entre images, s'avère prometteuse pour la réalisation d'un outil simplifié à la fois fonctionnel en milieu forestier et peu paramétrable. Divers tests sur d'autres blocs d'images acquis à basse altitude ont 


\begin{tabular}{rrrr}
\hline Modèle & $r^{2}$ aj. & RMSE $(\mathrm{m})$ & RMSE $(\%)$ \\
\hline$H$ dom $=\alpha+\beta * p 99$ & 0,82 & 1,68 & 8,5 \\
$H d o m=\alpha+\beta *$ mean $+\gamma *$ std & 0,82 & 1,65 & 8,4 \\
\hline
\end{tabular}

TABLE 1 : Modèles de régression à une et deux variables explicatives pour prédire la hauteur dominante $(H d o m)$ en peuplements feuillus irréguliers $(n=36)$. Les variables explicatives sont des statistiques synthétisant le modèle numérique de canopée photogrammétrique ( $p 99$ : $99^{\text {ième }}$ percentile; mean : moyenne; std : écart-type). Les performances des modèles sont exprimées en terme de coefficient de détermination ajusté ( $r^{2}$ aj.), d'erreur moyenne quadratique (RMSE) et d'erreur moyenne quadratique relative (RMSE \%).

confirmé que l'utilisation de la corrélation en géométrie images permet l'obtention de résultats plus robustes et de meilleure qualité que ceux en géométrie terrain, du moins sur des zones présentant des variations de relief du même ordre de grandeur que la hauteur de vol.

\subsection{Inventaire à deux phases de la hauteur domi- nante}

Les modèles de régression de prédiction de la hauteur dominante basés sur les indices de statistique descriptive du MNC photogrammétrique sont présentés table 1. Ces résultats mettent en évidence la capacité de prédire la hauteur dominante avec un degré de précision de l'ordre de 1.65 mètres (erreur moyenne quadratique), et ce pour des peuplements à structure irrégulière. Le degré d'irrégularisation des peuplements de Felenne est illustré à la figure 8, sur laquelle est tracée la limite d'une des 36 placettes d'inventaires.

L'inspection visuelle du MNC photogrammétrique pour chacune des placettes d'inventaire a mis en évidence un défaut dans la méthodologie d'inventaire. En effet, certaines couronnes d'arbre sont partiellement présentes sur le MNC de la placette d'inventaire alors que leur tige en elle-même n'est pas localisée dans la placette, et n'a donc pas fait l'objet de mesure. Dans le but de modéliser la relation entre le MNC et la hauteur dominante, il aurait été plus adéquat d'inventorier sur le terrain tous les arbres dont le houppier est présent dans la placette, peu importe que leur tronc le soit ou non. De cette manière, les indices statistiques du MNC calculés pour une placette donnée seraient exclusivement liés aux arbres mesurés sur le terrain. L'inventaire aurait donc du se faire "par le haut", à l'instar de la prise de vue aérienne.

$\mathrm{Au}$ vu de la performance des modèles présentés table 1 et pour peu que la stratégie d'acquisition soit optimisée dans ce but, on peut s'attendre à obtenir des résultats pour des peuplements réguliers qui soient d'une précision sub-métrique, c'est à dire du même ordre de grandeur que la mesure sur le terrain. De plus, l'utilisation de l'information spectrale pourrait permettre une discrimination des essences forestière, et améliorer ainsi la prédiction de la hauteur dominante. La constitution de série temporelle de MNCs permettrait dès lors de déduire les accroissements en hauteur des peuplements par photogrammétrie, et ainsi d'étudier la productivité à laquelle la hauteur dominante est étroitement liée. De telles investigations ont déjà été menées par le passé avec l'emploi d'images aéroportées grand format (voir Véga and StOnge (2009)), mais la technologie des drones permet de travailler avec plus de flexibilité à une résolution plus fine et à une échelle plus locale, ce qui répond aux besoins des scientifiques et gestionnaires actifs dans le domaine de la foresterie de précision.

\section{Remerciements}

Nos vifs remerciements vont premièrement aux pilotes de l'unité de Gestion des Ressources forestières et des Milieux naturels : Alain Monseur et Cédric Geerts. Les opérations d'inventaires ont été menées par Coralie Mengal et Frédérique Henrotay, à qui nous adressons également nos remerciements. Nous exprimons notre reconnaissance à la Direction Générale des Transports Aériens, représentée en la personne de Erika Billen, ainsi que la commune de Beauraing pour avoir délivré les autorisations nécessaires aux opérations avec un drone.

\section{Références}

Aber, J. S., Marzolff, I., Ries, J. B., 2010. Small-Format Aerial Photography : Principles, Techniques and Geoscience Applications. Elsevier Science \& Technology Books.

Anderson, K., Gaston, K. J., 2013. Lightweight unmanned aerial vehicles will revolutionize spatial ecology. Frontiers in Ecology and the Environment 11 (3), 138-146.

Assman, E., 1959. Höhenbonität und wirkliche ertragsleistung. Forstwiss. Centralbl. 78, 1-64.

Baltsavias, E., Gruen, A., Eisenbeiss, H., Zhang, L., Waser, L. T., 2008. High-quality image matching and automated generation of 3D tree models. International Journal of Remote Sensing 29 (5), 1243-1259.

Bonnet, S., Toromanoff, F., Bauwens, S., Michez, A., Dedry, L., Lejeune, P., 2013. Principes de base de la télédétection et ses potentialités comme outil de caractérisation de la ressource forestière - II. LiDAR aérien. Forêt Wallonne 124, 28 41.

Dandois, J. P., Ellis, E. C., 2013. High spatial resolution three-dimensional mapping of vegetation spectral dynamics using computer vision. Remote Sensing of Environment 136, 259-276.

EDF R\&D Telecom ParisTech, 2014. CloudCompare (version 2.4) [GPL software].

URL Retrieved from http://www.danielgm. net/cc/.

Koh, L. P., Wich, S. A., 2012. Dawn of drone ecology : lowcost autonomous aerial vehicles for conservation. Tropical Conservation Science 5 (2), 121-132. 

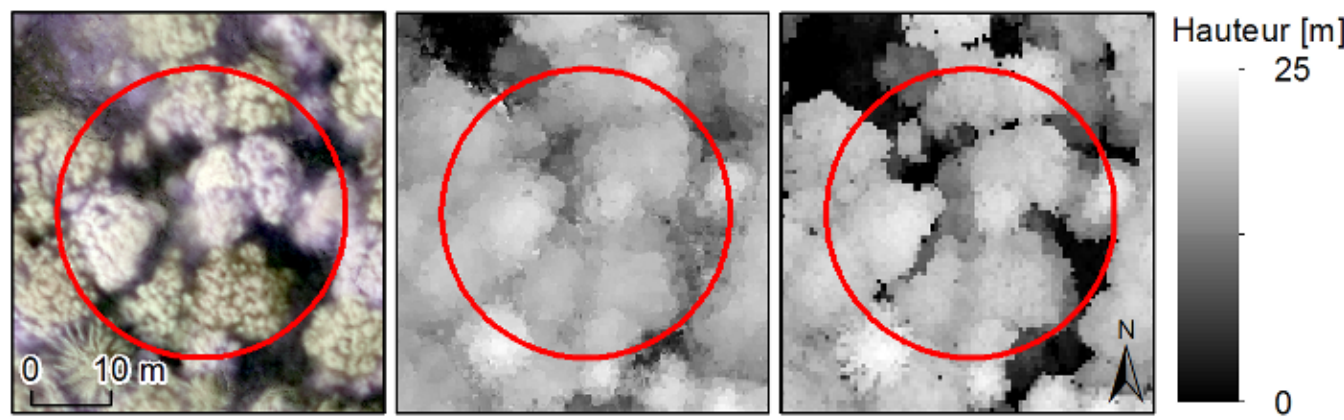

FIGURE 8 : MNC photogrammétrique généré avec l'outil MICMAC (figure au centre) centré sur une placette d'inventaire (cercle rouge). À gauche : une orthophotomosaique en fausse couleur. À droite : le MNC LiDAR. Les niveaux de gris représentent la hauteur de la canopée [m].

Lejeune, P., Gheysen, T., Rondeux, J., 2008. Les inventaires forestiers en futaie feuillue : Quelles méthodes pour quels objectifs ? Forêt Wallonne 93, 16-24.

Lisein, J., Pierrot-Deseilligny, M., Bonnet, S., Lejeune, P., 2013. A photogrammetric workflow for the creation of a forest canopy height model from small unmanned aerial system imagery. Forests 4 (4), 922-944.

Lowe, D., 2004. Distinctive image features from scale-invariant keypoints. International Journal of Computer Vision 60 (2), 91-110.

Næsset, E., 2002. Predicting forest stand characteristics with airborne scanning laser using a practical two-stage procedure and field data. Remote Sensing of Environment 80 (1), 88-99.

Otoul, C., 1978. Contribution à l'étude de la productivité du chêne rouvre en ardenne (région de wellin). Annales de Gembloux 4 (4), 237-248.

Paparoditis, N., Baghli, I., Thom, C., 2000. Reconstruction de modèles numériques de surfaces en zone urbaine à partir de prises de vues aériennes numériques à fort recouvrement. Bulletin d'Information de l'IGN 3 (71), 27-33.

Pénard, L., Paparoditis, N., Pierrot-Deseilligny, M., 2006. Reconstruction 3D automatique de façades de bâtiments en multi-vues. In : Actes de la Conférence Reconnaissance des Formes et Intelligence Artificielle. Tours, France.

Pierrot-Deseilligny, M., 2014. Micmac documentation : MicMac, Apero, Pastis and Other Beverages in a Nutshell!

URL http://logiciels.ign.fr/?-Micmac,3-

Pierrot-Deseilligny, M., Clery, I., 2011a. Apero, an open source bundle adjustment software for automatic calibration and orientation of set of images. The International Archives of the Photogrammetry, Remote Sensing and Spatial Information Sciences XXXVIII-5/W16, 1-8.

Pierrot-Deseilligny, M., Clery, I., 2011b. Évolutions récentes en photogrammétrie et modélisation $3 \mathrm{~d}$ par photo des milieux naturels. Collection EDYTEM 12, 51-64.

Pierrot-Deseilligny, M., Paparoditis, N., 2006. A multiresolution and optimization-based image matching approach : An application to surface reconstruction from SPOT5-HRS stereo imagery. The International Archives of the Photogrammetry, Remote Sensing and Spatial Information Sciences 36.

[R] Development Core Team, 2011. R : A Language and Environment for Statistical Computing. R Foundation for Statistical Computing, Vienna, Austria, ISBN 3-900051-07-0.

URL http://www.R-project.org/

Rondeux, J., 1999. La mesure des arbres et des peuplements forestiers (deuxième édition). Les Presses agronomiques de Gembloux, Gembloux.

Snavely, N., Seitz, S. M., Szeliski, R., Nov. 2008. Modeling the world from internet photo collections. International Journal of Computer Vision 80 (2), 189-210.

Véga, C., St-Onge, B., 2008. Height growth reconstruction of a boreal forest canopy over a period of 58 years using a combination of photogrammetric and lidar models. Remote Sensing of Environment 112 (4), 1784 - 1794, remote Sensing Data Assimilation Special Issue.

Véga, C., St-Onge, B., 2009. Mapping site index and age by linking a time series of canopy height models with growth curves. Forest Ecology and Management 257 (3), 951 - 959.

Watts, A. C., Ambrosia, V. G., Hinkley, E. A., 2012. Unmanned aircraft systems in remote sensing and scientific research : Classification and considerations of use. Remote Sensing 4 (6), 1671-1692.

White, J., Wulder, M., Vastaranta, M., Coops, N., Pitt, D., Woods, M., Jun. 2013. The utility of image-based point clouds for forest inventory : A comparison with airborne laser scanning. Forests 4 (3), 518-536. 